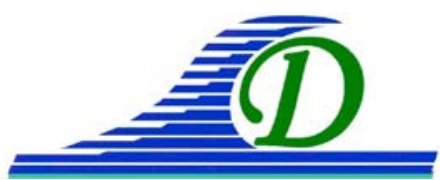

XIII İ̀es Journées Nationales Génie Côtier - Génie Civil

Dunkerque, 2-4 juillet 2014

DOI:10.5150/jngcgc.2014.084 C Editions Paralia CFL

disponible en ligne - http://www.paralia.fr - available online

\title{
Simulation numérique d'un parc pilote d'hydroliennes avec des vitesses de courant extrêmes : application au cas du Raz Blanchard
}

\author{
Van Thinh NGUYEN ${ }^{1}$, Sylvain GUILLOU ${ }^{1}$, Alina SANTA CRUZ ${ }^{1}$ \\ 1. Laboratoire Universitaire des Sciences Appliquées de Cherbourg, EA 4253, \\ Rue Louis Aragon, BP 78, 50130 Cherbourg-Octeville, France. \\ van-thinh.nguyen@unicaen.fr ; sylvain.guillou@unicaen.fr ; \\ alina.santa-cruz@unicaen.fr
}

\section{Résumé :}

L'effet sur la puissance disponible des fermes hydroliennes dépend de nombreux facteurs, entre autres la vitesse du courant, le rendement de la machine et pour un parc l'espacement entre les machines. Cet article présente une méthodologie pour étudier l'écoulement au sein d'un parc d'hydroliennes. Les hydroliennes sont représentées par le concept de l'Actuator Disk. Une validation est réalisée sur la base des expériences de HARRISON et al. (2010) pour une machine. Puis, un parc de 10 turbines est étudié dans les conditions d'écoulement correspondant à la vitesse maximale atteinte pendant un cycle de marée de vives eaux.

Mots-clés : Parc pilote, Hydroliennes, Simulation Numérique, Actuator Disk.

\section{Introduction}

Les développements dans les énergies marines renouvelables se sont accrus au cours des dix dernières années en réponse à l'épuisement des ressources d'énergies fossiles et aux effets de serre induits par leur combustion. Les hydroliennes ont une part à jouer dans la diversification de l'énergie. Les sites d'intérêt industriels actuels sont dénombrables. L'un de ces sites est le Raz Blanchard. Il est situé entre l'île d'Aurigny et la côte ouest du Cotentin. Les courants, très forts, atteignent lors des marées exceptionnelles des vitesses de l'ordre de $5 \mathrm{~m} \mathrm{~s}^{-1}$. Ce site recense à lui seul $50 \%$ du potentiel national (BENBOUZID et al., 2011). Cela a conduit le gouvernement à sélectionner ce site pour lancer un appel à projet pour le déploiement de fermes pilotes de 4 à 10 hydroliennes (ONEM, 2013).

L'étude du sillage proche est importante pour optimiser le fonctionnement d'une machine (BELHACHE et al., 2012 ; BELHACHE et al., 2014). A cette échelle locale, les équations de Navier-Stokes sont résolues avec un maillage fin permettant de représenter la géométrie de la machine. A l'échelle de la ferme hydrolienne, l'optimisation du positionnement des machines nécessite de simuler précisément le sillage lointain ce qui permet d'estimer la capacité de l'écoulement à récupérer sa vitesse pour une machine située à l'aval. A cette échelle, les machines sont 
généralement représentées par le saut de pression qu'elles engendrent lors de la captation de l'énergie. C'est la base du modèle de l'Actuator Disk (ROC et al., 2013 ; MYERS \& BAHAJ, 2010) qui consiste à ajouter un terme source simulant les forces d'extractions d'une machine.

Dans cet article, nous validons un modèle utilisant le concept de l'Actuator Disk avec les résultats expérimentaux de HARRISON et al. (2010). Les résultats sont en très bonne correspondance, notamment dans le sillage lointain. Nous simulons ensuite un parc pilote de 10 machines, de 10 mètres de diamètre et étudions l'écoulement au sein de ce parc. Les machines sont disposées en quinconce. Les conditions d'écoulement correspondent à des vitesses de courant extrêmes, soit la vitesse maximale atteinte lors d'une marée de vives eaux dans le Raz Blanchard.

\section{Méthode}

\subsection{Théorie de l'Actuator Disk.}

Dans la théorie de l'Actuator Disk, le rotor de la turbine est assimilé à un disque homogène induisant une discontinuité de pression lorsqu'il est traversé par le courant. La force induite permet l'extraction de l'énergie cinétique de l'écoulement (MULUGETA BIADGO et al., 2013) et une réduction de la vitesse en aval du rotor. Cette force est donnée par l'équation (1) en fonction d'une part de la surface $S$ du disque et d'autre part du saut de pression $\Delta P$ qui dépend de la vitesse en amont du disque $U_{\infty}$, de la masse volumique du fluide $\rho$ et du coefficient de poussée $C_{T}$. La valeur de $C_{T}$ dans cette équation est discutée dans MYERS \& BAHAJ, (2010) et CASTELLANI \& VIGNAROLI, (2013). Selon la limite de Betz la valeur maximale de ce coefficient est de $8 / 9$.

$$
F_{t}=S \Delta P=\frac{1}{2} C_{T} \rho S U_{\infty}^{2}
$$

\subsection{Equations de base et démarche}

L'effort induit par le disque sur le fluide est introduit comme un terme source $S_{i}$ dans les équations de Navier-Stokes stationnaires :

$\frac{\partial\left(\rho U_{i} U_{j}\right)}{\partial x_{j}}=-\frac{\partial p}{\partial x_{i}}+\frac{\partial}{\partial x_{j}}\left[\mu\left(\frac{\partial U_{i}}{\partial x_{j}}+\frac{\partial U_{j}}{\partial x_{i}}\right)+R_{i j}\right]+\rho g_{i}+S_{i}$

$\frac{\partial U_{i}}{\partial x_{i}}=0 \quad$ (Notation d'Einstein)

$$
R_{i j}=\mu_{t}\left(\frac{\partial U_{i}}{\partial x_{j}}+\frac{\partial U_{j}}{\partial x_{i}}\right)
$$

Où $U_{i}$ sont les composantes de la vitesse, $x_{i}$ sont les coordonnées du point, $\mu$ est la viscosité dynamique moléculaire de l'eau, $R_{i j}$ est le tenseur de Reynolds lié à la vitesse par l'hypothèse Boussinesq, $\mu_{t}=C_{\mu} k^{2} / \varepsilon$ est la viscosité dynamique turbulente calculée à partir de l'énergie cinétique de la turbulence $k$ et de la dissipation $\varepsilon$, et $g$ est 


\section{XIII $I^{\text {èmes }}$ Journées Nationales Génie Côtier - Génie Civil \\ Dunkerque, 2-4 juillet 2014}

l'accélération gravitationnelle. Les termes sources ne sont appliqués que sur les éléments de maillage représentant la zone de la turbine. Il s'agit d'une densité volumique de force liée à la force de résistance du disque :

$S_{i}=\frac{F_{t}}{S e}=\frac{1}{2} \rho \frac{K}{e} U_{d}^{2}$

Où $K$ est un coefficient de résistance et $e$ est l'épaisseur du disque. Ceci s'appuie sur la méthode proposée par HARRISON et al. (2010). En considérant l'intérieur du disque comme un milieu poreux le coefficient de résistance est lié au coefficient de poussée $C_{T}$ par (TAYLOR, 1963) :

$C_{T}=\frac{K}{(1+0,25 K)^{2}}$

Pour un coefficient $C_{T}$ de 0.89 , le coefficient de résistance $K$ sera de 2, valeur utilisée par la suite. Le calcul de l'écoulement est tridimensionnel et stationnaire. Il est réalisé avec le code commercial ANSYS FLUENT 14.5. Le modèle de turbulence $k$-epsilon standard est utilisé.

\subsection{Validation de la méthode}

Nous aborderons dans un premier temps le cas d'une seule hydrolienne. Les résultats numériques sont confrontés aux résultats obtenus expérimentalement par HARRISON et al. (2010), pour un disque poreux dans un écoulement en canal. Le canal mesure $5 \mathrm{~m}$ de long et $1,36 \mathrm{~m}$ de large. La hauteur d'eau est de $0,3 \mathrm{~m}$. Le disque a un diamètre $D=0,1 \mathrm{~m}$ et une épaisseur $e=0,001 \mathrm{~m}$. Il est situé à 20 diamètres à l'aval de la section d'entrée du canal et à 30 diamètres en amont de la section de sortie. Les résultats expérimentaux ont été obtenus par vélocimétrie acoustique Doppler par MYERS et al. (2010), les mesures ont été obtenues sur des échantillons de 3 minutes d'acquisition avec une fréquence de $50 \mathrm{~Hz}$. Les auteurs estiment la précision de leurs mesures à $1 \%$. Le domaine de calcul est couvert avec un maillage hexaédrique de 16867840 éléments avec un raffinement dans la zone du disque (la taille des mailles dans cette zone est égale à l'épaisseur du disque e). Des conditions de symétrie sont imposées sur les bords latéraux et au niveau de la surface libre. Au fond, une condition d'adhérence est appliquée. En sortie, la pression statique est imposée, tandis qu'en entrée un profil de vitesse $U_{I}(z)$ et un profil d'intensité I de l'énergie cinétique turbulente $k$ sont imposés (voir figure 1) avec les relations (6).

$U_{I}(z)=2,5 U^{*} \ln \left(\frac{z U^{*}}{v}\right)+A ; \quad k=\frac{3}{2} I^{2} U^{2} ; \quad \varepsilon=C_{\mu}^{3 / 4} \frac{k^{1 / 2}}{l}$

Où la vitesse de frottement est égale à $U^{*}=0,00787 \mathrm{~m} \mathrm{~s}^{-1}$, la constante vaut $A=0,197 \mathrm{~m} \mathrm{~s}^{-1}, z$ est la cote verticale, $v$ est la viscosité cinétique, $U$ est la vitesse moyenne, $C_{\mu}=0,09$, et $l$ est la longueur caractéristique des tourbillons (ici la profondeur de l'eau). 

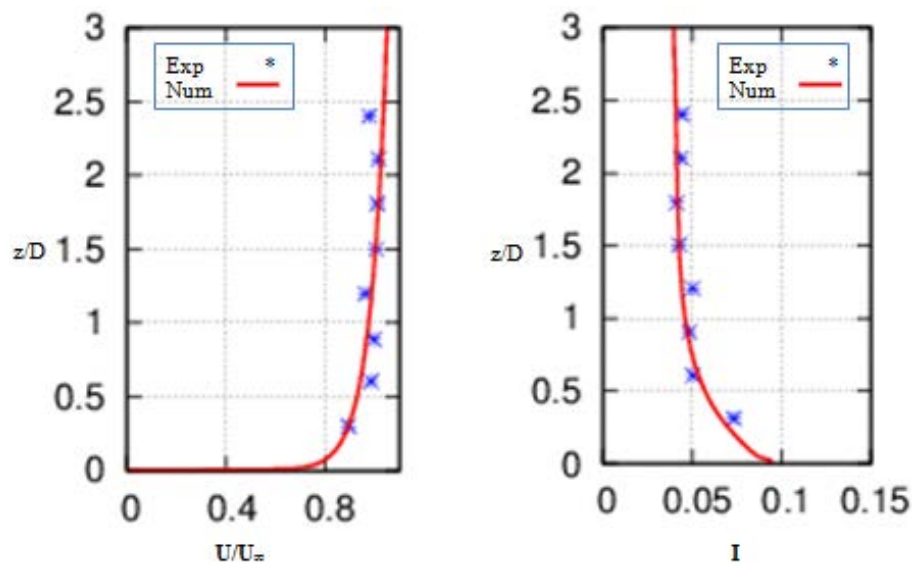

Figure 1. Profils verticaux de vitesse axiale (gauche) et d'intensité turbulente à l'entrée du canal (droite). Comparaison des résultats numériques (Num) et expérimentaux (Exp) de HARRISON et al. (2010).

Le long de l'axe du disque, la distribution de la vitesse et de l'intensité turbulente (voir figure 2) indique que le sillage lointain $(x>8 D)$ est bien simulé contrairement au sillage proche. En dessous de $8 D$, la vitesse est assez bien reproduite mais l'intensité turbulente est sous-estimée. La figure 3 montre les profils verticaux de vitesse et d'intensité turbulente. Les profils numériques coïncident avec les résultats expérimentaux, surtout dans le sillage lointain (voir figures 3c, 3d et 3e). Notons que le long de l'axe de la machine $(z / D=1,5)$, l'erreur relative maximale sur la vitesse est de $21 \%$ dans le sillage proche et de $2 \%$ dans le sillage lointain (voir figures 3a1 et 3e1). Pour l'intensité turbulente, les erreurs sont de $62 \%$ et $6 \%$ pour les sillages proche et lointain respectivement (voir figures 3a2 et 3e2).

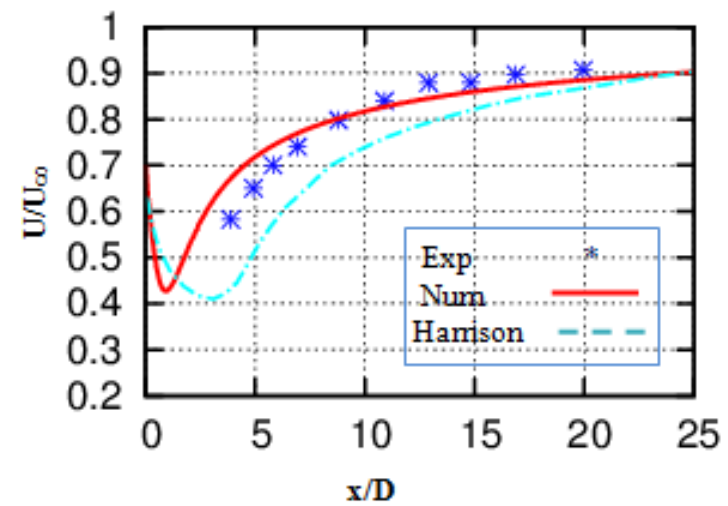

(a)

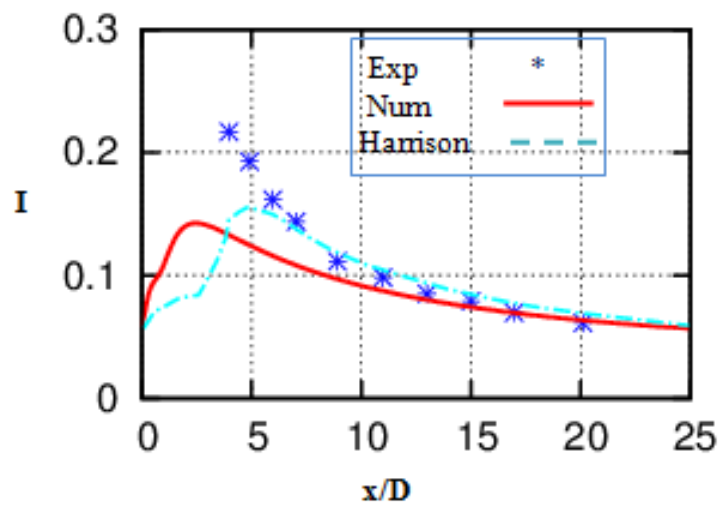

(b)

Figure 2. Profils à l'aval du disque ( $x=0$ correspond à la position du disque), le long de l'axe central. Comparaison des résultats numériques (Num), expérimentaux (Exp) et numérique (Harrison) de HARRISON et al. (2010).

a) vitesse longitudinale (adimensionnée par $\left.U_{\infty}\right)$, b) intensité turbulente. 


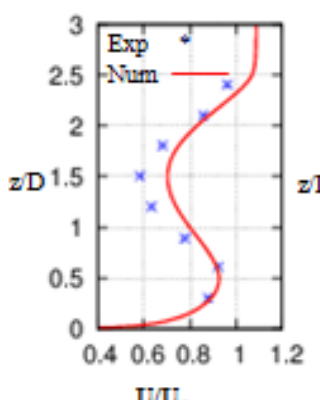

(al)

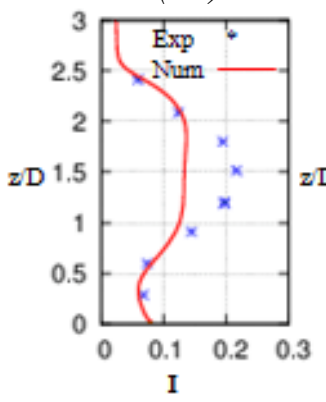

(a2)

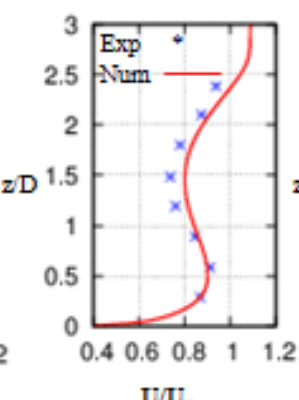

(b1)

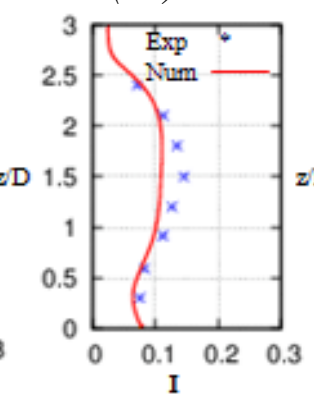

(b2)

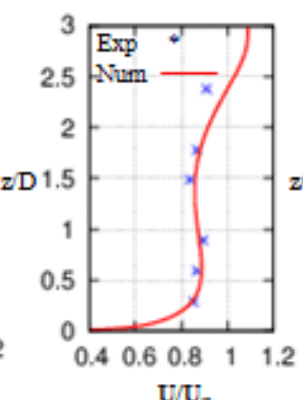

(c1)

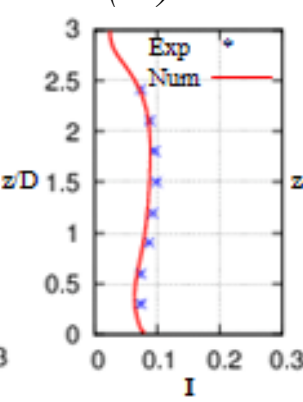

(c2)

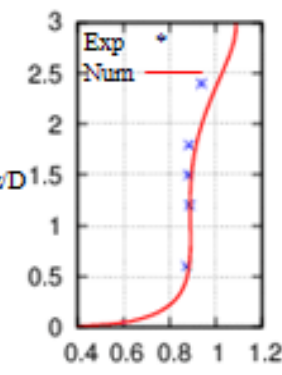

$\mathrm{U} / \mathrm{U}_{\circ}$

(d1)

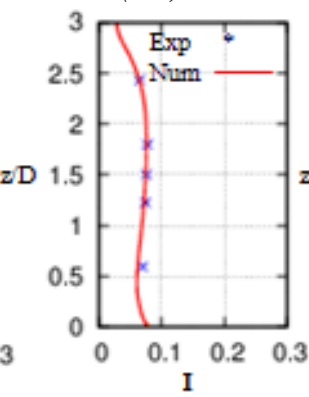

(d2)

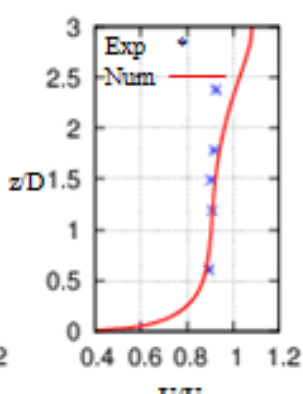

$\mathrm{U} / \mathrm{U}$

(e1)

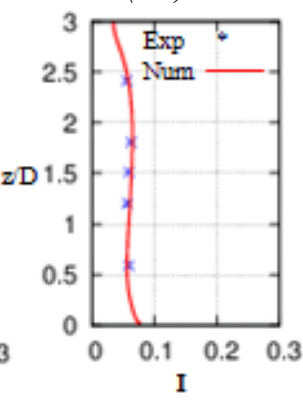

(e2)

Figure 3. Profils verticaux de vitesse longitudinale (adimensionnée par $U_{\infty}$ )(haut) et d'intensité turbulente (bas), à l'aval du disque, le long de l'axe central. Comparaison des résultats numériques (Num) et expérimentaux (Exp) de HARRISON et al., 2010). $x$ est la distance à l'aval du disque : a) $x=4 D, b) x=7 D$, c) $x=11 D$, d) $x=15 D$ et e) $x=20 D$.

HARRISON et al. (2010) ont relevé le fait que le modèle de l'Actuator Disk ne reproduisait pas bien l'écoulement dans le sillage proche du disque perforé $(<5 D)$. La raison principale est que l'écoulement dans le sillage proche est fortement instationnaire à des échelles plus petites. Ces effets doivent dont être pris en compte par le modèle de turbulence. Ici, un modèle de turbulence $k$ - $\varepsilon$ classique a été utilisé. Il n’intègre donc pas la génération ou la dissipation de la turbulence due au passage du disque (TEDDS et al., 2014). HARRISON et al. (2010) ont fait des simulations semblables avec un modèle $k$ - $\omega$ SST. Ils obtiennent des résultats similaires dans le sillage proche (voir figure 2). On notera cependant que le rétablissement de la vitesse est retardé dans leur simulation. La vitesse dans le sillage est plus éloignée des résultats expérimentaux alors que l'intensité de la turbulence est mieux approchée. Il en ressort que l'utilisation de l'Actuator Disk avec un modèle de turbulence de type $k-\varepsilon$ donne des bons résultats pour le sillage lointain et permet d'estimer la longueur de rétablissement.

\section{Simulation d'un parc pilote}

La méthode est appliquée à un parc pilote de 10 hydroliennes avec des conditions de courant extrêmes (vitesse maximale de l'écoulement lors d'une marée de vives eaux) et une hauteur d'eau de $50 \mathrm{~m}$. Nous caractérisons ainsi le déficit de vitesse, le saut de 
pression et les sillages produits en aval des machines en tenant compte de l'interaction entre les machines.

L'étude porte sur un parc pilote de 10 hydroliennes. Le domaine de calcul est de $600 \mathrm{~m}$ de long, $400 \mathrm{~m}$ de large avec une hauteur d'eau de $50 \mathrm{~m}$. Les turbines sont toutes identiques de diamètre $D=10 \mathrm{~m}$ et d'épaisseur e=2 m. Elles sont disposées en quinconce (BAI et al., 2009). Trois lignes de turbines sont distribuées dans le domaine (voir figure 4). La première ligne est située à 20 diamètres à l'aval de la section d'entrée et la dernière ligne est située au minimum à 10 diamètres en amont de la section de sortie. Le domaine de calcul est couvert avec un maillage hexaédrique de 12162492 éléments. Il y a un raffinement dans les zones de turbines. La taille des mailles dans ces zones est inférieure à l'épaisseur du disque $(e)$. Le rapport entre l'épaisseur du disque et la taille de maille selon l'axe $x$ (respectivement $y$ ) est de 20 (respectivement 5). Nous reprenons les conditions du cas de validation: profil de vitesse et profil d'énergie cinétique turbulente donnés par l'équation (6) ; conditions sur les parois latérales, sur la surface, au fond et la pression statique en sortie du canal. Nous modifions la vitesse maximale en entrée $U_{\infty}=3,5 \mathrm{~m} \mathrm{~s}^{-1}$, la vitesse de frottement $U^{*}=0,1294 \mathrm{~m} \mathrm{~s}^{-1}$, la constante $A=-1,57 \mathrm{~m} \mathrm{~s}^{-1}$. Deux espacements latéraux $a$ ( $3 D$ et $5 D$ ) (avec $2 a$ est la distance entre deux machines d'une même ligne) et trois espacements longitudinaux $b$ entre deux lignes de machines $(5 D, 10 D$ et $15 D)$ sont testés (voir figure 4).

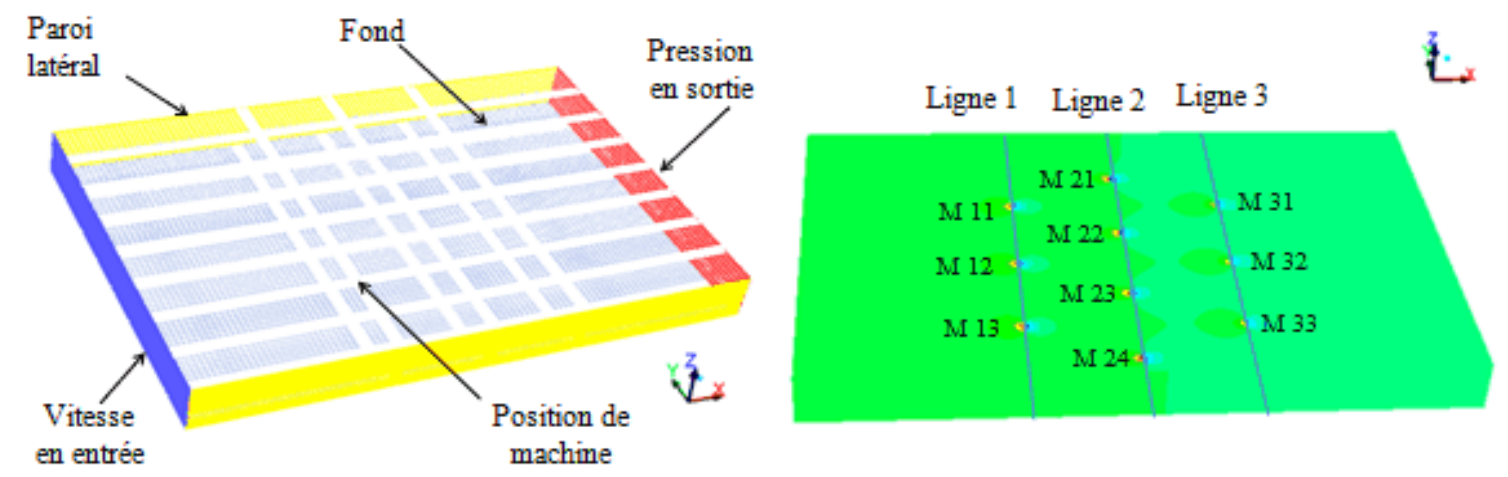

Figure 4. Domaine de calcul et position des machines dans un parc.

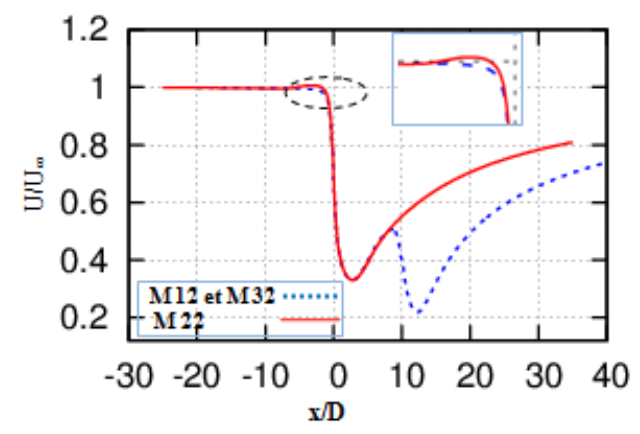

Figure 5. Profils axiaux de vitesse longitudinale dans le cas de référence

$$
(a=3 D \text { et } b=5 D) \text {. }
$$




\section{XIII $I^{\text {èmes }}$ Journées Nationales Génie Côtier - Génie Civil \\ Dunkerque, 2-4 juillet 2014}

La figure 5 présente les profils de vitesse longitudinale dans le cas de référence pour les machines M12 et M32 (qui appartiennent à deux lignes différentes mais ont même ordonnée $y$ ) et pour la machine M22. Pour M22 le profil a été recalé sur l'abscisse de la machine M12. Cette figure montre qu'il faut au moins une distance de $5 D$ après la turbine avant que la vitesse ne commence à augmenter. A la distance de $10 D, 50 \%$ de la vitesse d'entrée est récupéré. Pour atteindre 100\%, une distance de l'ordre de $40 \mathrm{D}$ semble nécessaire du fait d'un rétablissement asymptotique. Ces profils mettent en évidence que la distance latérale entre les machines affecte la vitesse de l'écoulement au niveau des machines de la ligne suivante. Ainsi une légère survitesse apparait en amont des machines de la seconde ligne. Cette survitesse est plus importante pour $a=3 D$ que pour $a=5 D$. Cependant, notons que cette accélération reste légère $(0,9 \%)$. La puissance cinétique (en $W$ ) d'un fluide traversant une machine de section $S$ est calculée par $P_{c i n}=0,5 \rho U_{\infty}{ }^{3}$. Nous utilisons cette définition pour calculer la puissance hydraulique disponible.

Tableau 1. Puissance moyenne par machine et par ligne (en $\mathrm{kW}$ ) pour les différents cas

\begin{tabular}{lllllll}
\hline $\boldsymbol{a}$ & $\mathbf{3 D}$ & $\mathbf{3 D}$ & $\mathbf{3 D}$ & $\mathbf{5 D}$ & $\mathbf{5 D}$ & $\mathbf{5 D}$ \\
$\boldsymbol{b}$ & $\mathbf{5 D}$ & $\mathbf{1 0 D}$ & $\mathbf{1 5 D}$ & $\mathbf{5 D}$ & $\mathbf{1 0 D}$ & $\mathbf{1 5 D}$ \\
\hline Ligne 1 & 1378 & 1378 & 1378 & 1378 & 1378 & 1378 \\
Ligne 2 & 1409 & 1423 & 1426 & 1399 & 1412 & 1413 \\
Ligne 3 & 179 & 439 & 624 & 164 & 432 & 621 \\
\hline
\end{tabular}

Tableau 2. Puissance pour chaque machine de la deuxième ligne (en $\mathrm{kW}$ ) pour les configurations avec $a=3 D$.

\begin{tabular}{llll}
\hline $\boldsymbol{a}$ & 3D & 3D & 3D \\
$\boldsymbol{b}$ & $\mathbf{5 D}$ & $\mathbf{1 0 D}$ & $\mathbf{1 5 D}$ \\
\hline$M 21$ & 1404 & 1416 & 1418 \\
$M 22$ & 1414 & 1430 & 1433 \\
$M 23$ & 1414 & 1430 & 1433 \\
$M 24$ & 1404 & 1416 & 1418 \\
\hline
\end{tabular}

Ainsi, le tableau 1 présente les valeurs de la puissance moyenne par machine et par ligne pour les diverses configurations étudiées tandis que le tableau 2 présente un zoom sur les machines de la seconde ligne. Il s'ensuit l'évaluation de l'impact des espacements longitudinal $a$ et transversal $b$ sur les puissances disponibles pour les lignes de machines placées en aval.

Quelle que soit la configuration (voir tableau 1), le potentiel énergétique reste inchangé pour les machines de la première ligne, alors qu'un léger effet bénéfique de l'effet de blocage sur l'écoulement apparaît pour la deuxième ligne. Le tableau 2 nous permet de faire un zoom à l'intérieur de la deuxième ligne. L'accélération de l'écoulement observée entre les machines amont a un impact sur la puissance disponible pour les 
machines placées au centre (M22 et M23). Elle est légèrement supérieure à celle des machines placées en extrémité (M21 et M24). En effet, pour la configuration ( $a=3 D$ et $b=5 D$ ) (respectivement $(a=3 D$ et $b=15 D)$ ) les machines centrales ont $0,94 \%$ (respectivement 1,07\%) de puissance disponible en plus par rapport aux machines placées en extrémité.

Quelle que soit la configuration (voir tableau 1), les fortes vitesses de courant, le rapport des puissances entre la première et la troisième ligne est très défavorable. La puissance moyenne par machine et par ligne est de l'ordre de 2,3\% de plus que pour la première ligne pour le cas de référence $(a=3 D$ et $b=5 D)$. Par contre, pour la troisième ligne la puissance disponible est très faible. En effet, pour le cas de référence elle ne dépasse pas $13 \%$ de la puissance de la première ligne. La variation de $a$ n'a qu'un faible impact sur les puissances de la deuxième et troisième ligne, ainsi, on observe une diminution de $0,7 \%$ entre le cas de référence $(a=3 D$ et $b=5 D)$ et le cas $(a=5 D$ et $b=5 D)$ pour la deuxième ligne et de $8,5 \%$ pour la troisième ligne.

La distance longitudinale entre la première et la troisième ligne est le paramètre critique $(2 b)$. Il correspond au rétablissement du potentiel énergétique de l'écoulement. L'augmentation de l'espacement $b$ permet de réduire le rapport de puissances entre la première et la troisième ligne. Ainsi, lorsque $b$ est égal à $15 D$ la troisième ligne présente $45 \%$ de la puissance de la première ligne au lieu de $13 \%$ pour le cas de référence $(b=5 D)$. En somme, l'augmentation de l'espacement $b$ permet une meilleure récupération des vitesses dans le sillage des machines de la première ligne.

Lorsque $b$ est grand, l'influence de l'espacement $a$, déjà faible, s'estompe (pour $b=15 D$ le rapport des puissances disponibles entre la troisième et la première ligne reste de l'ordre 45\%).

\section{Conclusion}

Le modèle de l'Actuator Disk a été utilisé pour modéliser un parc de 10 machines. La méthode a été validée sur la base de résultats expérimentaux de la littérature en ce qui concerne la distance de rétablissement de l'écoulement à l'aval du disque. Des questions restent posées quant à la modélisation de la turbulence dans le proche sillage. Sans un terme supplémentaire dans le modèle de turbulence, le modèle $k$ - $\varepsilon$ standard semble fournir une meilleure estimation de la longueur de rétablissement que le modèle $k$ - $\omega$ SST. Ceci a conduit à utiliser le premier pour l'étude du parc pilote de 10 machine. Il ressort des simulations du parc que l'espacement longitudinal (2b) entre deux lignes de turbines parfaitement alignées est le paramètre clé si l'on est en présence d'un courant unidirectionnel.

Cependant, la modélisation de la turbulence dans le sillage proche par modification de modèles de type $k-\varepsilon$ continue à faire débat (KASSMI \& MASSON, 2008) et reste une voie d'amélioration possible. L'écoulement dans le Raz Blanchard est orienté sud-ouest nord -est en moyenne. Cependant, il existe un écart d'angle par rapport à cette direction 


\section{XIII $I^{\text {èmes }}$ Journées Nationales Génie Côtier - Génie Civil \\ Dunkerque, 2-4 juillet 2014}

qui peut aller jusqu'à $30^{\circ}$ par endroit. Aussi, des études complémentaires doivent intégrer cette variation. De plus la variation temporelle du courant de marée fait que la technique présentée doit être adaptée pour simuler un cycle de marée complet. Enfin, la nature particulièrement accidentée des zones à fort courant comme le Raz Blanchard impose de mener des études complémentaires sur l’impact de la bathymétrie.

\section{Remerciements}

Les auteurs remercient le gouvernement vietnamien pour le financement de la thèse de Van Thinh NGUYEN et le Syndicat Mixte du Cotentin pour le financement des moyens de calcul. Ils remercient également Jérôme THIEBOT pour ses conseils avisés.

\section{Références bibliographiques}

BAI L., SPENCE R.R.G., DUDZIAK G. (2009). Investigation of the Influence of Array Arrangement and Spacing on Tidal Energy Converter (TEC) Performance using a 3Dimensional CFD Model. Proceedings of the 8th European Wave and Tidal Energy Conference, Uppsala, Sweden.

BELHACHE M., GUILLOU S., MOUAZE D., SANTA CRUZ A., GRANGERET P. (2012). Etude numérique de l'intégration d'hydroliennes (à axe vertical) en courant giratoire. Journées Nationales Génie Côtier - Génie Civil, Cherbourg, pp 819-826. http://dx.doi.org/10.5150/jngcgc.2012.089-B

BELHACHE M., GUILLOU S., GRANGERET P., MOUAZE D., SANTA CRUZ A. (2014). Wake numerical study of a vertical marine current turbine, La Houille Blanche, 2014 (sous presse).

BENBOUZID M., ASTOLFI J.A., BACHA S., CHARPENTIER J.F., MACHMOUM M., MAITRE T., ROYE D. (2011). Concepts, modélisation et commandes des hydroliennes. Energies marines renouvelables aspects généraux, éolien, marémoteur et hydrolien sous la direction de Bernard Multon. Lavoisier, Paris, Chap. 8, pp 265-328.

CASTELLANI F., VIGNAROLI F. (2013). An application of the actuator disc model for wind turbine wakes calculations. Applied Energy, Vol. 101, pp 432-440. http://dx.doi.org/10.1016/j.apenergy.2012.04.039

HARRISON M.E., BATTEN W.M.J., MYERS L.E., BAHAJ A.S. (2010). Comparison between CFD simulation and experiments for predicting the far wake of horizontal axis tidal turbines. IET Renew, Power Gener, Vol. 4, ISS 6, pp 613-627. http://dx.doi.org/10.1049/iet-rpg.2009.0193

KASMI A.E., MASSON C. (2008). An extended $k$ - $\varepsilon$ model for turbulent flow through horizontal-axis wind turbines. Journal of Wind Engineering and Industrial Aerodynamics, Vol. 96, pp 103-122. http://dx.doi.org/10.1016/j.jweia.2007.03.007 
Thème 5 - Énergies et ressources marines

MULUGETA BIADGO A., SIMONOVIC A., KOMAROV D., STUPAR S. (2013). Numerical and Analytical Investigation of Vertical Axis Wind Turbine. FME Transactions, Vol. 41, pp 49-58.

MYERS L.E., BAHAJ A.S. (2010). Experiment analysis of the flow field around horizontal axis tidal turbines by use of scale mesh disk rotor simulators. Ocean Engineering, Vol. 37, pp 218-227. http://dx.doi.org/10.1016/j.oceaneng.2009.11.004

ONEM -Ouest Normandie Energies Marines- (2013). La Basse-Normandie s'inspire du site écossais de l'EMEC pour les projets de fermes pilotes hydroliennes au large du Raz Blanchard. Communiqué de presse aux Iles Orcades, Ecosse, le 28 octobre 2013.

ROC T., CONLEY D.C., GREAVES D. (2013). Methodology for tidal turbine representation in ocean circulation model. Renewable Energy, Vol. 51, pp 448-464. http://dx.doi.org/10.1016/j.renene.2012.09.039

TAYLOR G.I. (1963). The scientific papers of Sir Geoffrey Ingram Taylor, ed. G.K. Batchelor. Cambridge University Press.

TEDDS S.C., OWEN I., POOLE R.J. (2014). Near-wake characteristics of a model horizontal axis tidal stream turbine, Renewable Energy, Vol. 63, pp 222-235. http://dx.doi.org/10.1016/.renene.2013.09.011 